\title{
SOLUÇÕES DE MANUTENÇÃO NA PÓS-COLHEITA DE Chrysanthemum morifolium cv. Dragon
}

\section{Chrysanthemum morifolium cv. Dragon postharvest maintenance solutions}

\author{
Poliana Cristina Spricigo ${ }^{1}$, Ben-Hur Mattiuz ${ }^{2}$, Júlia De Pietro ${ }^{3}$, \\ Claudia Fabrino Machado Mattiuz ${ }^{4}$, Maria Elisa Morais de Oliveira $^{5}$
}

\begin{abstract}
RESUMO
O desenvolvimento e uso de técnicas que prolonguem a durabilidade das flores, mantendo a qualidade do produto, é imprescindível para redução de perdas pós-colheita. Objetivou-se, neste trabalho, avaliar soluções de manutenção, associadas ou não a sacarose, na manutenção da qualidade pós-colheita de hastes de crisântemos. O experimento foi conduzido sob delineamento inteiramente casualizado, em esquema fatorial, com cinco tratamentos e três repetições, com 2 hastes florais cada uma. Os tratamentos utilizaram água destilada; 8-HQC a $100 \mathrm{mg} \mathrm{L}^{-1}$; 8-HQC a $100 \mathrm{mg} \mathrm{L}^{-1}+$ sacarose a $50 \mathrm{~g} \mathrm{~L}^{-1}$, 8-HQC a $200 \mathrm{mg} \mathrm{L}^{-1}$; 8-HQC a $200 \mathrm{mg} \mathrm{L}^{-1}+\mathrm{sacarose}^{-\mathrm{H}}$ a $50 \mathrm{~g} \mathrm{~L}^{-1}$. Foram feitas avaliações físicas: coloração, massa fresca e conteúdo relativo de água (CRA); avaliações químicas: açúcares redutores e pigmentos; e avaliações qualitativas: turgescência, cor das flores, e número de botões, flores entreabertas e abertas. A combinação de 8-HQC $200 \mathrm{mg} \mathrm{L}^{-1}+$ sacarose a $50 \mathrm{~g} \mathrm{~L}^{-1}$ foi a que apresentou melhor desempenho para a manutenção da qualidade das hastes florais, favorecendo a abertura de botões e a turgescência das lígulas. A sacarose contribuiu para uma melhor manutenção de substâncias de reserva na haste, que tiveram a sua vida de vaso estendida.
\end{abstract}

Termos para indexação: Crisântemo, 8-citrato de hidroxiquinolina, sacarose.

\begin{abstract}
The development and use of techniques that extend the life vase of the flowers, maintaining the quality of the product, is essential for reducing postharvest losses. The objective of this work was to evaluate different solutions for maintenance, associated or not to sucrose, in maintaining the postharvest quality of chrysanthemum stems. The treatments used distilled water, 8-HQC to $100 \mathrm{mg} \mathrm{L}^{-1}, 8$-HQC to $100 \mathrm{mg} \mathrm{L}^{-1}+$ sucrose $50 \mathrm{~g} \mathrm{~L}^{-1}$, 8-HQC to $200 \mathrm{mg} \mathrm{L}^{-1}, 8$-HQC to $200 \mathrm{mg} \mathrm{L}^{-1}+$ sucrose $50 \mathrm{~g} \mathrm{~L}^{-1}$. Physical assessments were made: color, fresh mass and relative water content; chemical evaluations: reducing sugars and pigments, and qualitative assessments: turgidity, color of the flowers, and number of buttons, open flowers and partially open flowers. The combination of 8-HQC $200 \mathrm{mg} \mathrm{L}^{-1}+$ sucrose $50 \mathrm{~g} \mathrm{~L}^{-1}$ was the best performance that made for maintaining the quality of flower stems, favoring the opening of buttons and turgidity of petals. Sucrose contributed to better maintenance of the reserve substances in the shaft, which had increased the flower vase life.
\end{abstract}

Index terms: Chrysanthemum, 8-hydroxyquinoline citrate, sucrose.

(Recebido em 16 de julho de 2009 e aprovado em 18 de fevereiro de 2010)

\section{INTRODUÇÃO}

O crisântemo de corte exerce extrema importância no mercado de flores no Brasil, devido à grande diversidade em cores e formas de inflorescências, assim como pela resposta precisa ao fotoperíodo. Entretanto, apesar dessas características, algumas cultivares de corte apresentam limitado período de conservação em vaso.

A senescência das flores e o amarelecimento de folhas são os principais sintomas da redução da qualidade, valor e vida de vaso de flores cortadas. O murchamento e a abscisão de flores são comuns em Alstroemeria, crisântemo e lírios cortados, e o amarelecimento das folhas são comuns em rosas miniaturas, poinsettia e crisântemo (Staby \& Erwin, 1977; Hibma, 1988; Tjosvold et al., 1994). Entre os compostos com ação microbiana, o 8hidroxiquinolina puro e seu éster, citrato (8-HQC), tem sido amplamente utilizado e podem duplicar ou triplicar a longevidade das flores. Além de ser um bactericida e fungicida eficiente, o 8-HQC tem-se mostrado um excelente redutor do bloqueio fisiológico da haste (Rogers, 1973). O 8-HQC também afeta o balanço hídrico de flores cortadas (Mattiuz et al., 2005), sendo que parte desse efeito benéfico foi atribuída à ação do composto no fechamento dos estômatos (Stoddard \& Miller, 1962).

\footnotetext{
1Universidade Estadual de Campinas/UNICAMP - Faculdade de Engenharia Agrícola - Avenida Candido Rondon - 501 - Cidade Universitária Zeferino Vaz - 13083-875 - Barão Geraldo - Campinas, SP - polianaspricigo@feagri.unicamp.br

2Universidade Estadual Paulista/ UNESP - Departamento de Tecnologia - Jaboticabal, SP

3Universidade Estadual Paulista/ UNESP - Jaboticabal, SP

${ }^{4}$ Centro Universitário Moura Lacerda - Ribeirão Preto, SP

${ }^{1}$ Universidade Estadual de Campinas/UNICAMP - Campinas, SP
} 
Os açúcares atuam na manutenção dos processos fundamentais para prolongar a vida decorativa das flores cortadas, melhorando o balanço hídrico pela regulação da transpiração e pelo aumento da absorção de água (Nowak \& Rudnicki, 1990). O fornecimento de açúcar exógeno retarda a senescência das flores (Paulin, 1986), substituindo o carboidrato endógeno esgotado pela respiração (Nowak et al., 1991). O composto 8-hidroxiquinolina (8-HQC) é conhecido como um potente bactericida e fungicida, sendo os seus ésteres, citrato de 8-hidroxiquinolina (8-HQC) e sulfato de 8hidroxiquinolina (8-HQS), testados para a avaliação de suas ações nos microrganismos das soluções conservantes.

Objetivou-se, nesta pesquisa, avaliar soluções de manutenção, associadas ou não à sacarose, na manutenção da qualidade pós-colheita de hastes de crisântemos.

\section{MATERIAL E MÉTODOS}

Hastes florais de Chrysanthemum morifolium Ramat. cv. Dragon foram colhidas no ponto de colheita comercial e imediatamente transportadas a seco do município de Holambra até o Laboratório de Tecnologia dos Produtos Agrícolas da FCAV-UNESP, Campus de Jaboticabal-SP.

No laboratório, as flores com defeitos ou injúrias decorrentes do transporte foram descartadas. Em seguida, as hastes florais foram submetidas à toalete eliminando-se as folhas basais, e à padronização a $50 \mathrm{~cm}$ de altura mediante o corte das hastes dentro de recipientes com água destilada. Após a padronização, as flores foram pesadas e colocadas em frascos com $500 \mathrm{~mL}$ de solução (manutenção) contendo: água destilada; 8-HQC $100 \mathrm{mg} \mathrm{L}^{-1}$; 8-HQC $100 \mathrm{mg} \mathrm{L}^{-1}+$ sacarose a $50 \mathrm{~g} \mathrm{~L}^{-1}, 8$-HQC $200 \mathrm{mg} \mathrm{L}^{-1}$; 8-HQC $200 \mathrm{mg} \mathrm{L}^{-1}+$ sacarose a $50 \mathrm{~g} \mathrm{~L}^{-1}$.

$\mathrm{O}$ experimento foi conduzido em delineamento inteiramente casualizado, num esquema fatorial composto por dois fatores: cinco tratamentos e quatro datas de amostragem $(0 ; 4 ; 8 ; 12$ dias). Foram utilizadas três repetições com duas hastes florais cada uma, perfazendo um total de seis hastes por tratamento.

A avaliação das características qualitativas foi realizada periodicamente através de atribuição de notas (observação visual) às folhas e flores senescentes, conforme o critério a seguir. Turgescência: nota $4=$ flores e folhas túrgidas; nota 3 = levemente murcha (murchamento em até $25 \%$ das flores e folhas); nota $2=$ murcha (murchamento em $25-50 \%$ das flores e folhas); nota $1=$ muito murcha (murchamento em mais de 50\% das flores e folhas). Cor das flores: nota 4 = cor viva; nota $3=$ levemente desbotada; nota 2 = desbotada; nota 1 = muito desbotada. Número de botões , flores entreabertas e abertas: foram contadas a quantidade de botões, flores entreabertas e abertas a cada avaliação. A vida de vaso foi considerada encerrada quando $50 \%$ ou mais flores de uma haste/repetição apresentaramse sem atrativos comerciais.

Em relação aos aspectos físicos, a coloração foi determinada usando-se o reflectômetro Minolta CR 200b, conforme o sistema da CIE 1976 (Minolta, 1994), o que permitiu calcular a luminosidade (L), o ângulo de cor $\left(\mathrm{h}^{\circ}\right) \mathrm{e}$ a cromaticidade (C). Para avaliação da massa fresca as flores foram pesadas em balança analítica. Já o conteúdo relativo de água (CRA), foi obtido de acordo coma metodologia descrita por Kramer (1983), sendo que, nos crisântemos em estudo, foram retiradas 10 pétalas (lígulas) de cada flor, em cada tratamento. Em seguida, as pétalas foram pesadas e imersas em água destilada, para hidratação por um período de 4 horas. Após esse período, as pétalas foram colocadas em pesa-filtro com capacidade para $100 \mathrm{~mL}$, pesados e levados para estufa com circulação de ar forçada, a $70^{\circ} \mathrm{C}$, para secagem, o que permitiu o cálculo do conteúdo relativo de água, expresso em porcentagem.

Também foram feitas análises químicas dos açúcares redutores, conforme metodologia de Honda et al. (1982). Para determinação dos pigmentos foram retiradas amostras das partes florais (lígulas) do crisântemo, pesando 1g, para determinação do conteúdo de antocianina, segundo a metodologia descrita por Francis (1982), expressa em mg $100 \mathrm{~g}^{-1}$.

\section{RESULTADOS E DISCUSSÃO}

Conforme a Figura 1 verifica-se que houve diferenças na perda acumulada de massa fresca entre os tratamentos e ao longo do período de armazenamento. Observa-se ainda que, a variação acumulada na massa fresca foi maior para o tratamento com água destilada, e essa diferiu significativamente das demais soluções. $\mathrm{O}$ tratamento com solução de 8-HQC $200 \mathrm{mg} \mathrm{L}^{-1}$ + sacarose a $50 \mathrm{~g} \mathrm{~L}^{-1}$ foi o que proporcionou menor variação na massa fresca acumulada.

Anju et al. (1999) observaram que hastes de crisântemos tratadas com 8-HQS + sacarose tiveram a vida de vaso prolongada e menor perda de massa fresca. Nos resultados obtidos para Chrysanthemum morifolium por Hassan \& Schmidt (2005), verifica-se que a perda de massa fresca foi diminuída de acordo com o aumento na concentração de 8-HQS. A adição da sacarose não minimizou as perdas, levando ainda a um efeito negativo. O melhor tratamento avaliado por ele foi o de 8-HQS a $400 \mathrm{ppm}$.

Pelos dados apresentados na Tabela 1, verifica-se que os tratamentos diferiram significativamente entre si com relação ao conteúdo de antocianinas, demonstrando que a utilização do 8-HQC associado ou não à sacarose contribui para a manutenção dos pigmentos. Ao longo do armazenamento, verifica-se para esse parâmetro, um aumento 
nos teores de antocianina até o $12^{\circ}$ dia. Esse aumento dos teores pode ser devido à perda de turgescência das hastes ao longo do período, fazendo com que uma quantidade maior de material fosse amostrada para efetuar a análise.

Nos tratamentos em que foi adicionada sacarose às soluções, observa-se uma maior quantidade de carboidratos redutores presentes nas flores, com destaque para o tratamento 8-HQC $200 \mathrm{mg} \mathrm{L}^{-1}+$ sacarose a $50 \mathrm{~g} \mathrm{~L}^{-1}$ que foi significativamente maior em relação aos demais. Esse efeito é mais bem verificado ao longo do armazenamento quando as hastes absorveram os carboidratos fornecidos pela solução (na forma de sacarose) acumulando-os nas lígulas, preferencialmente na forma de glicose. Segundo Rogers (1973), o fornecimento de açúcares

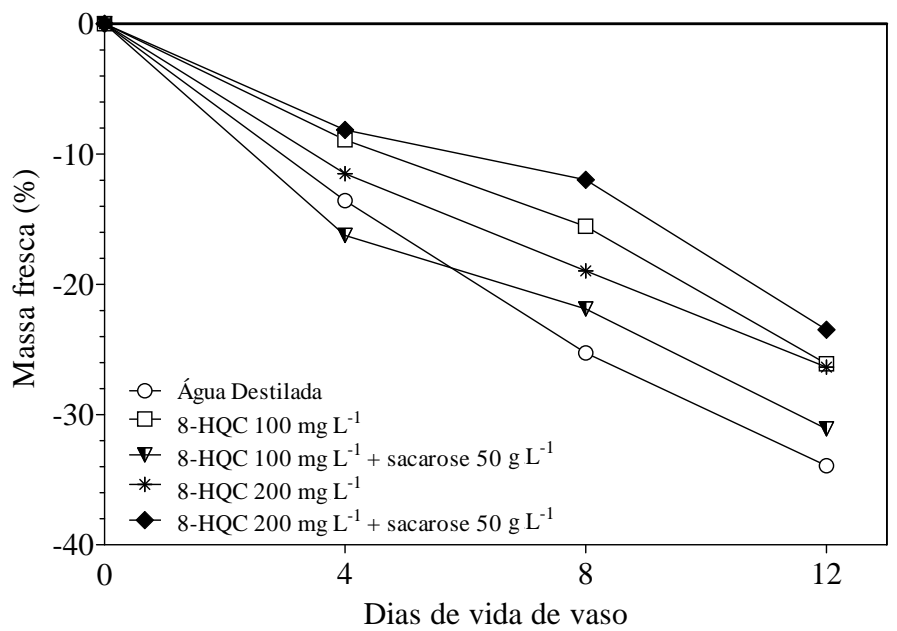

Figura 1 - Variação acumulada na massa fresca de hastes de crisântemo, submetidas a cinco soluções de tratamento e armazenadas por 12 dias a $22^{\circ} \mathrm{C}$.

Tabela 1 - Teor de antocianinas, carboidratos redutores e conteúdo relativo de água (CRA) de hastes de crisântemos submetidas a cinco soluções de manutenção e armazenadas por 12 dias a $22^{\circ} \mathrm{C}$.

\begin{tabular}{|c|c|c|c|}
\hline Variável & 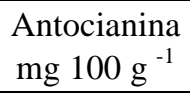 & $\begin{array}{l}\text { Carboidratos redutores } \\
\mathrm{g} \text { de glicose } 100 \mathrm{~g}^{-1}\end{array}$ & $\begin{array}{c}\text { CRA } \\
(\%) \\
\end{array}$ \\
\hline \multicolumn{4}{|l|}{ Tratamentos $(\mathrm{T})$} \\
\hline Água Destilada & $37,3 \mathrm{C}$ & $2,2 \mathrm{C}$ & $92,5 \mathrm{C}$ \\
\hline 8-HQC $100 \mathrm{mg} \mathrm{L}^{-1}$ & $41,0 \mathrm{~A}$ & $1,9 \mathrm{C}$ & $94,4 \mathrm{AB}$ \\
\hline 8-HQC $100 \mathrm{mg} \mathrm{L}^{-1}+$ sacarose a $50 \mathrm{~g} \mathrm{~L}^{-1}$ & $40,0 \mathrm{AB}$ & $2,9 \mathrm{~B}$ & $95,5 \mathrm{~A}$ \\
\hline 8-HQC $200 \mathrm{mg} \mathrm{L}^{-1}$ & $39,8 \mathrm{BC}$ & $2,6 \mathrm{C}$ & $94,9 \mathrm{AB}$ \\
\hline 8-HQC $200 \mathrm{mg} \mathrm{L}^{-1}+$ sacarose a $50 \mathrm{~g} \mathrm{~L}^{-1}$ & $38,8 \mathrm{BC}$ & $3,2 \mathrm{~A}$ & $93,8 \mathrm{BC}$ \\
\hline DMS & 1,73 & 0,37 & 1,52 \\
\hline \multicolumn{4}{|l|}{ Dias de vida no vaso (D) } \\
\hline 0 & $36,0 \mathrm{C}$ & $2,5 \mathrm{~A}$ & $92,0 \mathrm{C}$ \\
\hline 4 & $38,0 \mathrm{~B}$ & $2,6 \mathrm{~A}$ & $94,4 \mathrm{~B}$ \\
\hline 8 & $41,0 \mathrm{~A}$ & $2,8 \mathrm{~A}$ & $96,4 \mathrm{~A}$ \\
\hline 12 & $42,3 \mathrm{~A}$ & $2,6 \mathrm{~A}$ & $94,0 \mathrm{~B}$ \\
\hline DMS & 1,45 & 0,31 & 1,28 \\
\hline Interação TxD & $* *$ & $* *$ & $* *$ \\
\hline $\mathrm{CV}(\%)$ & 3,77 & 12,00 & 1,39 \\
\hline
\end{tabular}

Médias seguidas de pelo menos uma letra em comum, para cada variável, não diferem entre si pelo teste de Tukey, ao nível de 5\%, ** significativo a $\mathrm{P}=0,01$. DMS: diferença mínima significativa; C.V.: coeficiente de variação. 
na solução conservante, principalmente sacarose, é responsável pela reposição dos carboidratos consumidos pela respiração. Colocando hastes de Zantedeschia aethiopica (L.) Spreng. em soluções preservativas contendo 8-HQC + sacarose, Skutnik et al. (2004), observaram grandes perdas no conteúdo de açúcares redutores. As hastes tratadas com 8 -HQC $+2 \%$ sacarose perderam cerca de metade do seu conteúdo de carboidratos redutores iniciais quando chegaram ao dia 12 do experimento.

Ainda na Tabela 1 , mostra-se que houve diferenças significativas dos tratamentos em relação à testemunha, com relação ao conteúdo relativo de água, com exceção do tratamento 8-HQC a $200 \mathrm{mg} \mathrm{L}^{-1}+$ sacarose a $50 \mathrm{~g} \mathrm{~L}^{-1}$. Nos resultados da análise de variância, houve interação significativa entre as soluções de manutenção e os dias de avaliação para o conteúdo relativo de água (CRA) das hastes de crisântemo. O conteúdo relativo de água dessas hastes diminuiu ao longo dos dias de avaliação, em todos os tratamentos. Vale ressaltar que o tratamento com água destilada obteve a maior redução no CRA, demonstrando que somente o tratamento com água destilada não foi suficiente para garantir um balanço hídrico positivo ao longo dos dias de vida de vaso da cultivar de crisântemo Dragon.
Segundo Halevy (1976) a sacarose favorece o balanço hídrico das flores cortadas acumulando-se nas flores. Com isso, ocorre aumento da concentração de solutos osmoticamente ativos e, consequentemente a manutenção da turgescência das pétalas. Isso pode ser verificado de acordo com as notas atribuídas para turgescência das hastes avaliadas (Tabela 2). Verifica-se que as flores em solução contendo 8-HQC permaneceram mais túrgidas em comparação à água destilada. Esse efeito foi melhorado nas soluções que continham sacarose, destacando, assim, o potencial conservante dessas soluções. A turgescência é necessária para o desenvolvimento de botões florais, até a completa maturação, dando assim suporte à atividade metabólica da flor cortada.

De acordo com Kays (1991), o déficit hídrico pode resultar em descoloração das flores, redução de turgidez, aumento da suscetibilidade ao dano causado pelo frio e aceleração dos sintomas de senescência.

Para a variável cor, nota-se que as soluções de 8HQC, especialmente na concentração de $200 \mathrm{mg} \mathrm{L}^{-1}$, com adição de sacarose tiveram um desempenho melhor ao longo do armazenamento que nos demais tratamentos, mantendo a cor por mais tempo (Tabela 3). Observou-se

Tabela 2 - Turgescência em lígulas de crisântemos, de hastes submetidas a cinco soluções de manutenção e armazenadas por 12 dias a $22^{\circ} \mathrm{C}$.

\begin{tabular}{llccc}
\hline \multirow{2}{*}{ Soluções de Manutenção } & \multicolumn{3}{c}{ Dias de vida de vaso } \\
\cline { 2 - 5 } & 0 & 4 & 8 & 12 \\
\hline Água Destilada & 4 & 3 & 1 & 1 \\
8-HQC 100 mg L & 4 & 3 & 1 \\
8-HQC 100 mg L & 4 & 3 & 2 \\
8-HQC 200 mg L & 4 & 4 & 2 & 1 \\
8-HQC 200 mg L & & 3 & 2 \\
\hline
\end{tabular}

Critérios: 4 = flores e folhas túrgidas; 3 = levemente murcha; 2 = murcha; 1 = muito murcha.

Tabela 3 - Cor das hastes em lígulas de crisântemos, de hastes submetidas a cinco soluções de manutenção e armazenadas, por 12 dias a $22^{\circ} \mathrm{C}$.

\begin{tabular}{llllc}
\hline \multirow{2}{*}{ Soluções de Manutenção } & \multicolumn{3}{c}{ Dias de vida de vaso } \\
\cline { 2 - 4 } & 0 & 4 & 8 & 12 \\
\hline Água Destilada & 4 & 2 & 1 & 1 \\
8-HQC 100 mg L & 4 & 3 & 1 \\
8-HQC 100 mg L & & 2 & 1 \\
8-HQC 200 mg L & & 4 & 2 & 1 \\
8-HQC 200 mg L & & 4 & 2 \\
\hline
\end{tabular}

Critérios: 4 = cor viva; 3 = levemente desbotada; 2 = desbotada; 1 = muito desbotada. 
ao longo do período, um leve desbotamento da cor, passando de vermelha para uma tonalidade mais clara. Apesar dos resultados obtidos nas soluções com 8-HQC serem próximos, as flores submetidas às soluções sem o carboidrato e a testemunha apresentaram maior número de lesões necrosadas ao longo do período.
Almeida et al. (2008) verificou que não houve influencia do armazenamento e da associação de soluções conservantes na abertura de inflorescências de copo-de-leite. Isso vai de encontro ao presente trabalho, que houve evolução dos botões para flores entreabertas e, finalmente, para as flores abertas, de crisântemos (Figura 2). Dentre as soluções testadas,
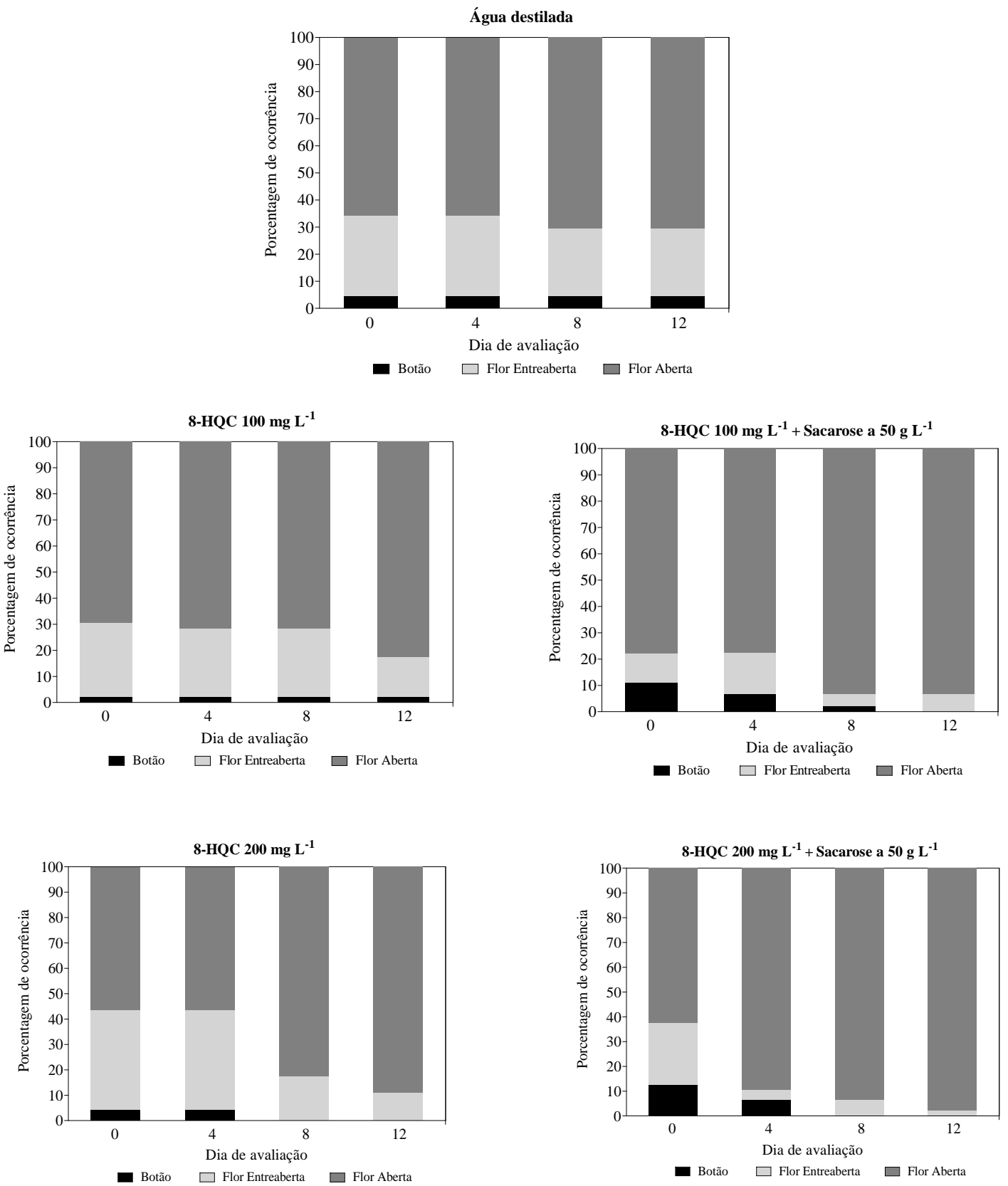

Figura 2 - Quantidade de botões, flores entreabertas e abertas durante os 12 dias que as hastes de crisântemos foram submetidas a cinco soluções de manutenção e armazenadas por 12 dias a $22^{\circ} \mathrm{C}$. 
os tratamentos com 8-HQC $200 \mathrm{mg} \mathrm{L}^{-1}$ e 8-HQC $200 \mathrm{mg} \mathrm{L}^{-1}+$ sacarose a $50 \mathrm{~g} \mathrm{~L}^{-1}$ proporcionaram a melhor evolução dos botões e das flores entreabertas para as flores abertas, com $57,7 \%$ e 56,7\%, respectivamente. Para Brackmann (2000), que armazenou flores em baixas temperaturas, o acréscimo de flores abertas do início ao final do armazenamento foi de aproximadamente $10 \%$. Nos resultados obtidos por Hassan \& Schmidt (2005) mostra-se que a utilização do 8-HQS em tratamentos para hastes de crisântemos, prolongou a vida de vaso e a qualidade das flores, independente de estar associado ou não à sacarose. À medida que a concentração de sacarose nas soluções aumentava, a resposta das flores para a abertura floral foi maior.

Ao analisar a coloração, observa-se que houve redução significativa para os três parâmetros observados (luminosidade, ângulo de cor e cromaticidade), para as flores de crisântemo, ao longo dos dias de vida de vaso (Tabela 4). A redução na luminosidade (onde $0=$ preto e 100 = branco), ao longo do período, pode ser atribuída ao fato das lígulas apresentarem-se com déficit hídrico e mostrarem sinais de lesões. Esse escurecimento também influenciou significativamente o ângulo de cor que intensificou a cor vermelha, durante o armazenamento. A redução da cromaticidade deve-se à degradação dos pigmentos presentes nas lígulas.

Também é possível observar diferenças significativas na coloração em relação aos tratamentos empregados, em que escurecimento das flores foi maior nas hastes colocadas apenas em água destilada (testemunha). Já o tratamento 8-HQC $200 \mathrm{mg} \mathrm{L}^{-1}+$ sacarose $50 \mathrm{mg} \mathrm{L}^{-1}$ demonstrou, estatisticamente, a melhor manutenção da luminosidade, com tendência de aumento nos valores observados ao longo do armazenamento. Verifica-se que a solução 8-HQC $200 \mathrm{mg} \mathrm{L}^{-1}+$ sacarose 50 $\mathrm{mg} \mathrm{L}^{-1}$ foi o tratamento que melhor manteve a cor vermelha, expressa pelo ângulo de cor, durante os dias de vida de vaso. Apesar de as hastes tratadas com sacarose apresentarem leve desbotamento de suas flores, a qualidade dessas foi superior a dos demais tratamentos devido à maior turgidez e ausência de pontos escurecidos. Para Mattiuz et al., (2005), o ângulo de cor não apresentou diferenças significativas, porém, o 1-MCP possibilitou melhor manutenção da cor vermelha em Alpinia purpurata (Vieill.) K. Schum. Houve redução da cor a partir do oitavo

Tabela 4 - Coloração em lígulas de crisântemos, de hastes submetidas a cinco soluções de manutenção e armazenadas por 12 dias a $22^{\circ} \mathrm{C}$.

\begin{tabular}{|c|c|c|c|}
\hline Variável & Luminosidade & Ângulo de cor & Cromaticidade \\
\hline \multicolumn{4}{|l|}{ Tratamentos $(\mathrm{T})$} \\
\hline Água Destilada & $24,5 \mathrm{C}$ & $10,8 \mathrm{~B}$ & $43,9 \mathrm{~A}$ \\
\hline 8-HQC $100 \mathrm{mg} \mathrm{L}^{-1}$ & $25,8 \mathrm{BC}$ & $11,9 \mathrm{AB}$ & $41,0 \mathrm{BC}$ \\
\hline 8-HQC $100 \mathrm{mg} \mathrm{L}^{-1}+$ sacarose a $50 \mathrm{~g} \mathrm{~L}^{-1}$ & $26,8 \mathrm{AB}$ & $12,4 \mathrm{AB}$ & $42,7 \mathrm{AB}$ \\
\hline 8 -HQC $200 \mathrm{mg} \mathrm{L}^{-1}$ & $25,6 \mathrm{BC}$ & $10,8 \mathrm{~B}$ & $40,7 \mathrm{BC}$ \\
\hline 8-HQC $200 \mathrm{mg} \mathrm{L}^{-1}+$ sacarose a $50 \mathrm{~g} \mathrm{~L}^{-1}$ & $28,3 \mathrm{~A}$ & $12,9 \mathrm{~A}$ & $43,9 \mathrm{~A}$ \\
\hline DMS & 1,68 & 1,78 & 2,04 \\
\hline \multicolumn{4}{|l|}{ Dias de vida no vaso (D) } \\
\hline 0 & $26,8 \mathrm{~A}$ & $13,5 \mathrm{~A}$ & $44,6 \mathrm{~A}$ \\
\hline 4 & $26,7 \mathrm{AB}$ & $12,3 \mathrm{~A}$ & $43,1 \mathrm{~A}$ \\
\hline 8 & $25,9 \mathrm{AB}$ & $10,8 \mathrm{~B}$ & $40,4 \mathrm{~B}$ \\
\hline 12 & $25,3 \mathrm{~B}$ & $10,8 \mathrm{~B}$ & $38,7 \mathrm{~B}$ \\
\hline DMS & 1,41 & 1,49 & 1,70 \\
\hline Interação TxD & n.s. & n.s. & $* *$ \\
\hline $\mathrm{CV}(\%)$ & 5,49 & 12,95 & 4,20 \\
\hline
\end{tabular}

Médias seguidas de pelo menos uma letra em comum, para cada variável, não diferem entre si pelo teste de Tukey, ao nível de 5\%, n.s., **, não significativo ou significativo a $\mathrm{P}=0,01$. DMS: diferença mínima significativa; C.V.: coeficiente de variação. 
dia de avaliação para todas as soluções avaliadas, com 1MCP tendo uma manutenção melhor da cor.

\section{CONCLUSÕES}

Nas soluções testadas de 8-HQC, a combinação de $200 \mathrm{mg} \mathrm{L}^{-1}+$ sacarose a $50 \mathrm{~g} \mathrm{~L}^{-1}$ foi a que apresentou melhor desempenho para a manutenção da qualidade das hastes florais, favorecendo a abertura de botões e a turgescência das lígulas. A sacarose contribuiu para melhor manutenção de substâncias de reserva na haste, que tiveram a sua vida de vaso estendida.

\section{REFERÊNCIAS BIBLIOGRÁFICAS}

ALMEIDA, E.F.A.; PAIVA, P.D.deO.; LIMA, L.C.deO.; RESENDE, M.L.; FONSECA, J.; TAVARES, T.S. Póscolheita de copo-de-leite: efeito de diferentes conservantes comerciais e armazenamento a frio. Ciência e Agrotecnologia, Lavras, v.32, n.4, p. 1189-1194, jul./ago., 2008.

ANJU, B.; TRIPATHI, S.N.; SEHGAL, O.P.; BHAT, A. Effect of pulsing, packaging and storage treatments on vase life of chrysanthemum cut flowers. Advances-inHorticulture-and-Forestry, Jodhpur, v.6, p.125-131, 1999.

BRACKMANN, A. Armazenamento de crisântemos Dedranthema grandiflora cv. Red Refocus em diferentes temperaturas e soluções conservantes. Revista Brasileira de Agrociência, Pelotas, v.6, n.1, p.19-23, 2000.

FRANCIS, F.J. Analysis of anthocyanins. In: MARKAKIS, P. (Ed.). Anthocyanins as food colors. New York: Academic, 1982. p.182-205.

HALEVY, A.H. Treatments to improve water balance of cut flowers. Acta Horticulturae, Palo Alto, v.64, p.223-230, 1976.

HASSAN, F.; SCHMIDT, G. Postharvest characteristics of cut carnations as the result of chemical treatments. Acta Agronomica Hungarica, Akadémiai Kiadó, v.52, n.2, p.125-132, 2005.

HIBMA, J.T. Development of a test for the control of the use of pre-treatment conditioning materials against leaf yellowing in Alstroemeria. Verslag Cêntrum voor Agrobiologisch, Onderzoek, v.91, p.26, 1988.

HONDA, S.; NISHIMURA, Y.; TAKAHASHI, M.; CHIBA, H.; KAKEHI, K. A manual method for spectrophotometric determination of reducing carbohydrates with 2-cyanoacetaminde. Annals of Chemistry, Ovidius, v.119, p.194-199, 1982.

KAYS, J.S. Postharvest physiology of perishable plant products. New York: V.N. Reinhold, 1991. 453p.

KRAMER, P.J. Water relations of plants. New York: Academic, 1983.489p.

MATTIUZ, C.F.M.; RODRIGUES, R. de J.D.; MATTIUZ, B.H.; PIVETTA, K.F.L. Physiological and qualitative aspects of postharvest conservation of red ginger [Alpinia purpurata (Vieill.) K. Schum.] inflorescences. Científica, Jaboticabal, v.33, n.1, p.83-90, 2005.

MINOLTA. Precise color communication: color control from feeling to instrumentation. Tokyo, 1994. 49p.

NOWAK, J.; GOSZCZYNSKA, M.D.; RUDNICKI, R.M. Storage of cut flowers and ornamental plants: present status and future prospects. Postharvest News and Information, v.2, n.4, p.255-260, 1991.

NOWAK, J.; RUDNICKI, R.M. Postharvest handling and storage cut flowers, florist greens and potted plants. Portland: Timber, 1990. 210p.

PAULIN, A. Influence of exogenous sugar on the evolution of some senescence petals. Acta Horticulturae, Noordwijkerhout, v.181, p.183-193, June 1986.

ROGERS, M.N. An historical and critical review of postharvest physiology research on cut flowers.

HortScience, Saint Joseph, v.8, n.3, p.189-194, 1973.

SKUTNIK, E.; RABIZA-WIDER, J.; WACHOWICZ, M.; LUKASZEWSKA, A.J. Senescence of cut leaves of Zantedeschia aethiopica and Z. elliottiana. Part I.

Chlorophyll degradation. ACTA Scientiarum

Polonorum, Hortorum Cultus, v.3, n.2, p.57-65, 2004.

STABY, G.L.; ERWIN, T.D. The storage of Easter lilies. Florists Review, Topeka, v.161, p.38, 1977.

STODDARD, E.M.; MILLER, P.M. Chemical control of water loss in growing plants. Science, Washington, n.137, p.224-225, 1962.

TJOSVOLD, S.A.; WU, M.; REID, M.S. Reduction of post production quality loss in potted miniature roses. HortScience, Saint Joseph, v.29, n.4, p.293-294, 1994 